hep-ph/9706392

UCLA/97/TEP/13

\title{
Two-Loop $\mathrm{N}=4$ Supersymmetric Amplitudes and QCD ${ }^{1}$
}

\author{
Zvi Bern², Joel Rozowsky and Bryce Yan \\ Department of Physics, UCLA, Los Angeles, CA 90095
}

\begin{abstract}
Two-loop four-gluon $N=4$ susy amplitudes are evaluated via cutting techniques as a testing ground for QCD. A conjecture for four-point amplitudes to all loop orders is described. We also present a new conjecture for the leading-color part of the two-loop five-gluon amplitudes.
\end{abstract}

\section{INTRODUCTION}

Over the years there have been a number of rather impressive Feynman diagram calculations at two and higher loops. (For one recent example see ref. [1]). However, a number of important computations remain to be performed. Two examples of computations which are required for analysis of experiments but have not been carried out are two-loop contributions to $e^{+} e^{-} \rightarrow 3$ jets and to Altarelli-Parisi splitting functions. More generally, no computations have appeared at two and higher loops which involve more than a single kinemtic variable.

In contrast, at one-loop, recent years have seen improvements in the calculational techniques for scattering amplitudes [2]. In this talk we discuss initial steps taken to apply some of these techniques to two- and higher-loop amplitudes. In particular, we apply the recent developments in cutting techniques for obtaining complete amplitudes with no ambiguities or subtractions. These cutting techniques have been used for obtaining infinite sequences of one-loop supersymmetric amplitudes [3] and for the calculations performed in refs. [4] of the one-loop helicity amplitudes for $e^{+} e^{-} \rightarrow 4$ jets and deeply inelastic scattering in association with three jets. Other approaches for efficiently calculating higher-loop amplitudes taken by various authors include string theory [5], first quantized [6] and recursive [7] approaches. (Further references are contained in ref. [2].)

A good starting point for exploring cutting techniques for higher-loops are $N=4$ supersymmetric amplitudes since they are particularly simple. Using cutting techniques we have obtained the exact expressions for the two-loop four-gluon $N=4$

1) This work was supported by the DOE under contract DE-FG03-91ER40662 and by the Alfred P. Sloan Foundation under grant BR-3222.

2) Presenter at 5th International Workshop on Deep Inelastic Scattering and QCD, April, 1997 
amplitudes [8]. Furthermore, with cutting techniques we have obtained conjectures for the structure of two-loop five-gluon amplitudes and for four-point amplitudes to arbitrary loop orders. We expect a study of $N=4$ amplitudes to be useful for obtaining two- and higher-loop QCD amplitudes via cutting techniques.

\section{PROPERTIES OF N=4 SUPERSYMMETRIC AMPLITUDES}

In performing the calculation we apply some of the techniques that have proven useful at tree level and at one loop. These include spinor helicity, color decompositions and supersymmetry identities. For references and further details the reader may consult various reviews $[9,2]$.

The high degree of supersymmetry present in $N=4$ amplitudes considerably simplifies their analytic structure. At one-loop the leading color $N=4$ partial amplitudes are rather simple and are given by [10]

$$
A_{4 ; 1}^{1 \text {-loop }}(1,2,3,4)=i s t A_{4}^{\text {tree }}(1,2,3,4) \mathcal{I}_{4}^{1 \text {-loop }}(s, t)
$$

where $\mathcal{I}_{4}^{1 \text {-loop }}(s, t)$ is the one-loop scalar integral and the tree amplitudes may be

found in ref. [9]. The Mandelstam variables are defined as $s \equiv\left(k_{1}+k_{2}\right)^{2}$ and $t \equiv\left(k_{2}+k_{3}\right)^{2}$. The massless $N=4$ super-multiplet consists of one gluon, four Weyl fermions and six real scalars, whose contributions are summed over to obtain the result (1). The subleading color contributions may be obtained in terms of sums of permutations of the expression (1).

When using cuts to obtain the two-loop $N=4$ gluon amplitudes we also need tree and one-loop amplitudes with either two external scalars or fermions, since these particles can also cross the cuts. The maximally helicity violating amplitudes with two external fermions or scalars may be obtained directly from the gluon amplitudes using supersymmetry identities.

\section{CONSTRUCTION OF TWO-LOOP AMPLITUDES}

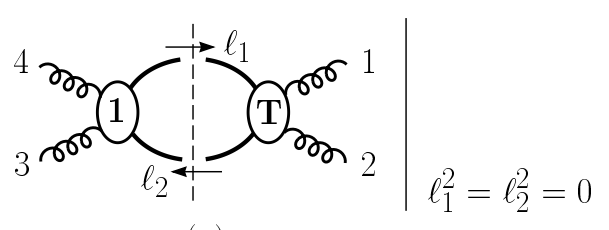

(a)

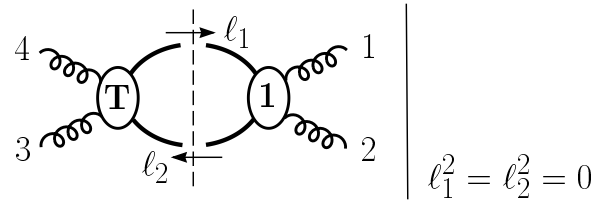

(b)

FIGURE 1. The two-particle $s$-channel cut has two contributions: one with the four-point one-loop amplitude ' 1 ' to the left and the tree amplitude ' $\mathrm{T}$ ' to the right (a) and the other being the reverse (b). 
A convenient way to obtain amplitudes is from cuts of unrestricted loop momentum integrals [2]. In this way, one may simultaneously construct the imaginary and real parts of the amplitudes. Consider for example, the cuts of the two-loop fourgluon amplitude. At two loops one must consider both two- and three-particle cuts and in each channel multiple cuts can contribute. For example, in Fig. 1 the two $s$ channel two-particle cuts are given. By combining all cuts into a single function, one obtains the full amplitude.

By computing cuts to higher orders in the dimensional regularization parameter $\epsilon=(4-D) / 2$ a complete reconstruction of a massless amplitude is possible $[11,2]$. This follows from dimensional analysis, since every term in an amplitude must have a prefactor of powers of $\left(-s_{i j}\right)^{-\epsilon}$ (where $s_{i j}=\left(k_{i}+k_{j}\right)^{2}$ ), which necessarily has cuts. For reasons of technical simplicity, we use helicity amplitudes which implicitly take the cut lines to have four-dimensional momenta. In ref. [8] we argued that for the two-loop four-point $N=4$ amplitudes this does not introduce any errors at least through $\mathcal{O}\left(\epsilon^{0}\right)$. However, for QCD amplitudes one would need to compute the contributions from the $(-2 \epsilon)$-dimensional parts of of the loop momenta, since they may interfere with poles in $\epsilon$ to produce $\mathcal{O}\left(\epsilon^{0}\right)$ contributions.

Applying the cut-construction method to the leading color part of the two-loop $N=4$ four-gluon partial amplitude, we obtain

$$
A_{4 ; 1 ; 1}^{\mathrm{LC}}=-s t A_{4}^{\mathrm{tree}}\left(s \mathcal{I}_{4}^{\mathrm{P}}(s, t)+t \mathcal{I}_{4}^{\mathrm{P}}(t, s)\right) .
$$

where $\mathcal{I}_{4}^{\mathrm{P}}(s, t)$ corresponds to the first $\phi^{3}$ scalar integral in Fig. 2 and $\mathcal{I}_{4}^{\mathrm{P}}(t, s)$ to the second.

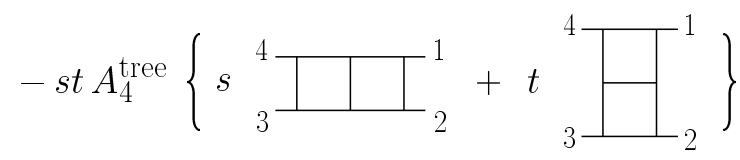

FIGURE 2. The result for the leading color two-loop amplitude, corresponding to Eq. (2). The diagrams correspond to scalar $\phi^{3}$ integrals.

The computation of the subleading color pieces is similar to the leading color computation. The complete two-loop four-gluon amplitudes may be found in ref. [8].

Following the same cut construction procedure used for the two-loop amplitudes, we have obtained a pattern for $n$-loop $N=4$ four-gluon amplitudes: one takes each $n$-loop graph in the $n$-loop amplitude and generates all the possible $(n+1)$-loop graphs by inserting a new leg between each possible pair of internal legs. Diagrams where triangle or bubble subgraphs are created should not be included. One also includes an additional factor of $i\left(\ell_{1}+\ell_{2}\right)^{2}$ in the numerator, where $\ell_{1}$ and $\ell_{2}$ are the momenta flowing through each of the legs to which the new line is joined, as depicted in Fig. 3. Each distinct $(n+1)$-loop graph should be counted once, even though they can be generated in multiple ways. The $(n+1)$-loop amplitude is then the sum of all distinct $(n+1)$-loop graphs multiplied by the tree amplitude. 


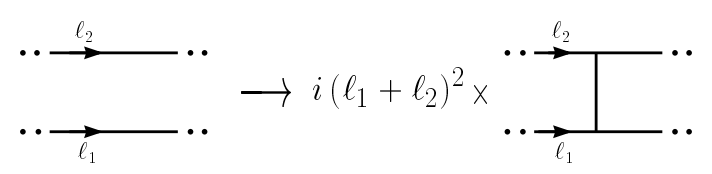

FIGURE 3. Starting from an $n$-loop integral function we may add an extra line to obtain results for $(n+1)$-loop amplitudes. The two-lines on the left represent two lines buried in some $n$-loop integral.

We have verified that this pattern is consistent with two-particle cuts to all loop orders and with four-dimensional three-particle cuts, up to five loops. The twoparticle cuts are not difficult to check because the same algebra appears at any loop order. The pattern remains a conjecture because we have not verified the higher-particle cuts. The same pattern also forms a conjecture for subleading in color contributions.

These methods may also be applied to higher-point amplitudes. In Fig. 4 we present a conjecture for the leading-color part of five-point two-loop $N=4$ amplitudes. The momentum $q$ appearing in the coefficients of the diagrams are loop momenta which should be integrated over. The complete consistency of this conjecture has not been proven.

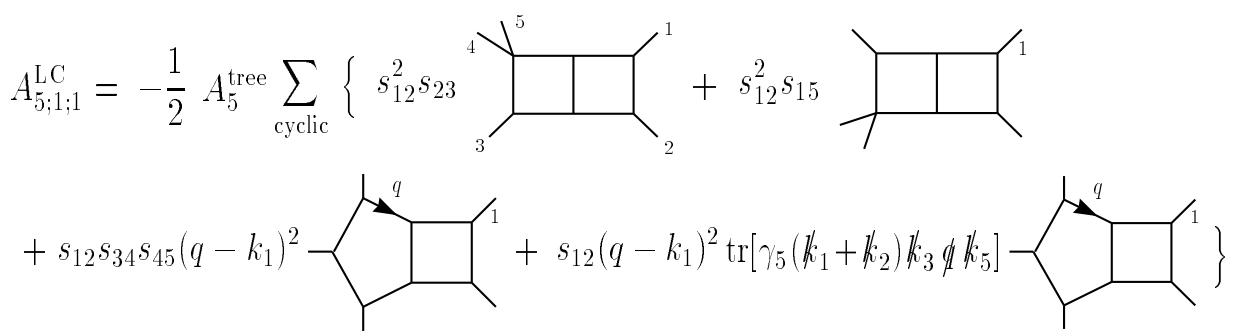

FIGURE 4. A conjecture for the leading color parts of the two-loop five-gluon amplitude. The arrow marks the line carrying loop momentum $q$.

The equivalence between leading log contributions of the one-loop $N=4$ amplitudes and FKL amplitudes [12] has recently been demonstrated [13]. We comment that the conjecture for the four-point multi-loop amplitudes presented here also has the expected behavior. Indeed, carrying out the leading log approximation on the conjecture in Fig. 3 yields,

$$
A_{4}^{\text {tree }}\left(\frac{s}{-t}\right)^{\alpha(t)}, \quad \alpha(t)=2 g^{2} N_{c} \frac{1}{\epsilon} \frac{1}{(4 \pi)^{2-\epsilon}} \frac{\Gamma(1+\epsilon) \Gamma^{2}(1-\epsilon)}{\Gamma(1-2 \epsilon)}\left(\frac{\mu^{2}}{-t}\right)^{\epsilon} .
$$

\section{CONCLUSIONS}

In this talk we outlined the initial steps that have been taken in applying the cut construction technique reviewed in ref. [2] to two-loop amplitudes. We expect 
that the cutting methods discussed here will be applied to two-loop QCD amplitudes. However, before physics can be extracted one would need numerically stable expressions for loop integrals and for the infrared divergent corners of phase space.

We thank L. Dixon and D.A. Kosower for many helpful discussions. We also thank V. Del Duca and C. Schmidt for notes on the relationship of $N=4$ and FKL amplitudes.

\section{REFERENCES}

1. T. van Ritbergen, in these proceedings.

2. Z. Bern, L. Dixon, and D.A. Kosower, Ann. Rev. Nucl. Part. Sci. 46:109 (1996), hep-ph/9602280; D.A. Kosower, in these proceedings.

3. Z. Bern, L. Dixon, D.C. Dunbar and D.A. Kosower, Nucl. Phys. B425:217 (1994), hep-ph/9403226; Nucl. Phys. B435:59 (1995), hep-ph/9409265.

4. Z. Bern, L. Dixon and D.A. Kosower, Nucl. Phys. Proc. Suppl. 51C:243 (1996), hepph/9606378; Z. Bern, L. Dixon, D.A. Kosower and S. Weinzierl, Nucl. Phys. B489:3 (1997), hep-ph/9610370; A. Signer and L. Dixon, Phys. Rev. Lett. 78:811 (1997), hep-ph/9609460; preprint hep-ph/9706285; Z. Bern, L. Dixon and D.A. Kosower, SLAC-PUB-7529.

5. L. Magnea, in these proceedings.

6. M.G. Schmidt and C. Schubert, Phys. Rev. D53:2150 (1996), hep-th/9410100; preprint hep-th/9611045; M. Reuter, M.G. Schmidt and C. Schubert, preprint hepth/9610191

7. G.D. Mahlon, Phys. Rev. D49:2197 (1994), hep-ph/9311213; Phys. Rev. D49:4438 (1994), hep-ph/9312276; C. Kim and V.P. Nair preprint hep-th/9608156

8. Z. Bern, J.S. Rozowsky and B. Yan, preprint hep-ph/9702424, to appear in Phys. Lett. B.

9. M. Mangano and S.J. Parke, Phys. Rep. 200:301 (1991); L. Dixon, preprint hepph/9601359, in Proceedings of Theoretical Advanced Study Institute in Elementary Particle Physics (TASI 95), ed. D.E. Soper.

10. M.B. Green, J.H. Schwarz and L. Brink, Nucl. Phys. B198:474 (1982).

11. W.L. van Neerven, Nucl. Phys. B268:453 (1986); Z. Bern and A.G. Morgan, Nucl. Phys. B467:479 (1996), hep-ph/9511336.

12. E.A. Kuraev, L.N. Lipatov and V.S. Fadin, Zh. Eksp. Teor. Fiz. 71:840 (1976) [Sov. Phys. JETP 44:443 (1976)].

13. V. Del Duca, in these proceedings. 